\title{
Complex kinetics and residual structure in the thermal unfolding of yeast triosephosphate isomerase
}

\author{
Ariana Labastida-Polito', Georgina Garza-Ramos², Menandro Camarillo-Cadena', Rafael A. Zubillaga' \\ and Andrés Hernández-Arana ${ }^{1 *}$
}

\begin{abstract}
Background: Saccharomyces cerevisiae triosephosphate isomerase (yTIM) is a dimeric protein that shows noncoincident unfolding and refolding transitions (hysteresis) in temperature scans, a phenomenon indicative of the slow forward and backward reactions of the native-unfolded process. Thermal unfolding scans suggest that no stable intermediates appear in the unfolding of yTIM. However, reported evidence points to the presence of residual structure in the denatured monomer at high temperature.
\end{abstract}

Results: Thermally denatured yTIM showed a clear trend towards the formation of aggregation-prone, $\beta$-strand-like residual structure when $\mathrm{pH}$ decreased from 8.0 to 6.0, even though thermal unfolding profiles retained a simple monophasic appearance regardless of $\mathrm{pH}$. However, kinetic studies performed over a relatively wide temperature range revealed a complex unfolding mechanism comprising up to three observable phases, with largely different time constants, each accompanied by changes in secondary structure. Besides, a simple sequential mechanism is unlikely to explain the observed variation of amplitudes and rate constants with temperature. This kinetic complexity is, however, not linked to the appearance of residual structure. Furthermore, the rate constant for the main unfolding phase shows small, rather unvarying values in the $\mathrm{pH}$ region where denatured yTIM gradually acquires a $\beta$-strand-like conformation. It appears, therefore, that the residual structure has no influence on the kinetic stability of the native protein. However, the presence of residual structure is clearly associated with increased irreversibility.

Conclusions: The slow temperature-induced unfolding of yeast TIM shows three kinetic phases. Rather than a simple sequential pathway, a complex mechanism involving off-pathway intermediates or even parallel pathways may be operating. $\beta$-strand-type residual structure, which appears below $\mathrm{pH} 8.0$, is likely to be associated with increased irreversible aggregation of the unfolded protein. However, this denatured form apparently accelerates the refolding process.

\section{Background}

It is now accepted that many proteins fold and unfold following complex kinetic models [1]. The most detailed kinetic studies of conformational change have been performed on small monomeric proteins by means of rapid mixing or fast temperature jumps, because protein molecules of this sort usually unfold reversibly but with

\footnotetext{
* Correspondence: aha@xanum.uam.mx

'Área de Biofisicoquímica, Departamento de Química, Universidad Autónoma Metropolitana-Iztapalapa, San Rafael Atlixco 186, Iztapalapa D.F. 09340, Mexico

Full list of author information is available at the end of the article
}

relaxation times ranging from less than a millisecond to a few seconds [2-4]. Previous studies have demonstrated the presence of transiently populated intermediates, apart from the native and unfolded end-states [1,3]. Intermediate states may be found either on- or off-pathway, and their interconnections may even result in the consolidation of parallel, competing folding-unfolding pathways $[5,6]$. Furthermore, the combination of experimental studies and molecular dynamics simulations has provided detailed structural descriptions of the multiple intermediates and transition states involved [7]. Recently, strong emphasis has been placed on the structural characterization of 
unfolded states, because the presence of residual, nativelike structure in parts of an otherwise unfolded polypeptide chain may be implicated in the speed of folding, as well as in the formation of misfolded molecules [8, 9].

However, there are examples of proteins that show very slow unfolding-refolding kinetics in the transition region (i.e., under conditions where the native and unfolded states are both significantly populated at equilibrium). Specifically, when unfolding is promoted by adding $\mathrm{GuHCl}$ or urea, slow-unfolding proteins take days to weeks to equilibrate, whereas for fast-unfolding proteins under similar conditions, equilibrium is reestablished in just a few hours [10-12]. Thus, if incubation times in the denaturing agent are not long enough, a slow-unfolding protein would display noncoincident unfolding and refolding profiles as the concentration of denaturing agent is varied (hysteresis). Likewise, hysteresis has been nicely demonstrated in the temperature-induced transitions of at least four proteins: an immunoglobulin light chain (monomer) [13], the Lpp-56 three-stranded $\alpha$-helical coiled coil [14], and two dimeric triosephosphate isomerases $[15,16]$. In these cases, thermal transitions detected by circular dichroism (CD) appear to be consistent with a two-state model with no intermediates.

Regarding trisosephosphate isomerase (TIM), many mesophilic members belonging to this enzyme family have been found to unfold slowly in chemical-denaturation studies, with one or more equilibrium or kinetic intermediates $[11,17,18]$. In contrast, thermal unfolding transitions of TIMs (in the absence of chemical denaturants) usually manifest themselves as monophasic profiles (i.e., simple sigmoidal curves with no evidence of intermediates), as recorded by CD [15, 19-21]. Unfortunately, irreversibility appears as a common feature in thermal unfolding, which has precluded the study of TIM refolding in cooling scans. Nevertheless, Benítez-Cardoza et al. [15] demonstrated that yeast TIM (yTIM) thermal unfolding is highly reversible at low protein concentration $(\approx 0.20 \mu \mathrm{M})$, although the unfolding-refolding cycle displays marked hysteresis when a heating-cooling rate of $2.0^{\circ} \mathrm{C} \mathrm{min}^{-1}$ is used. Attempts to achieve near-equilibrium transition profiles by decreasing the scan rate led to pronounced irreversibility [15].

At a fixed temperature, kinetic data for yTIM unfolding registered by far-UV circular dichroism (CD) in a restricted time span, are well-fitted by single exponential curves, whereas near-UV CD and fluorescence indicate biphasic kinetics. Refolding data are consistent with a second-order reaction [15]. Unlike yeast TIM, the enzyme from Trypanosoma cruzi (TcTIM) shows completely irreversible, temperature-induced denaturation, even at low protein concentration. Kinetic studies of this protein found that denaturation is a complex process in which two or three phases are clearly seen [19]. A common finding for both yTIM and TcTIM is that their denatured states appear to conserve some kind of residual structure based on calorimetric data $[15,19]$.

This work mainly focuses on determining the kinetic characteristics of temperature-induced yTIM unfolding in aqueous solution over long durations and in a wide $\mathrm{pH}$ range. Regardless of $\mathrm{pH}$, three kinetic phases were observed, although the small-amplitude faster phase was detected at only low temperatures. The relative amplitudes of the second and third phases vary with temperature in a way that seems difficult to explain by a sequential mechanism. The results thus evidence that the kinetics of yTIM thermal unfolding is more complex than previously thought. Furthermore, residual secondary structure was found in denatured yTIM below $\mathrm{pH}$ 8.0. Because this residual structure appears to be associated with the loss of refolding ability, its presence may indicate that misfolded, aggregation-prone structures are formed at high temperature. Molecular dynamics simulations showed that yTIM has a tendency to suffer $\alpha$-to- $\beta$ transitions when unfolded at high temperature, but this method does not properly reproduce the marked effect of $\mathrm{pH}$ on the structure of the thermally unfolded protein.

\section{Materials}

Overexpresion and purification of wild-type Saccharomyces cerevisiae TIM (yTIM) was carried out as described elsewhere [22]. Mass spectrometry (Additional file 1) and SDS-PAGE showed that the obtained enzyme was homogeneous. Enzymatic activity was determined by the coupled assay with $\alpha$-glycerophosphate dehydrogenase ( $\alpha$ GDH), using D-glyceraldehyde 3-phosphate (DGAP) as the TIM substrate [23]. Assays were performed at $25.0{ }^{\circ} \mathrm{C}$ in $1.0 \mathrm{~mL}$ of $0.1 \mathrm{M}$ triethanolamine buffer ( $\mathrm{pH} 7.4$ ) containing $10 \mathrm{mM}$ EDTA, $0.20 \mathrm{mM} \mathrm{NADH}, 0.02$ of $\alpha-\mathrm{GDH}$, and $2.0 \mathrm{mM}$ DGAP; the reaction was started by the addition of $3.0 \mathrm{ng}$ of yTIM, and NADH oxidation was followed by the change in absorbance at $340 \mathrm{~nm}$. The catalytic efficiency $\left(k_{\mathrm{cat}} / K_{\mathrm{M}}\right)$ of this enzyme was $5.0 \mathrm{x}$ $10^{6} \mathrm{~s}^{-1} \mathrm{M}^{-1}$, a value similar to that reported previously $[24,25]$.

\section{Circular dichroism spectra}

Circular dichroism (CD) spectra were obtained with a JASCO J-715 instrument (Jasco Inc., Easton, MD) equipped with a Peltier-type cell holder for temperature control and stirring with a magnetic bar. Cells of $1.00-\mathrm{cm}$ path length were used to keep the protein concentration near $10 \mu \mathrm{g} \mathrm{m}^{-1}(0.19 \mu \mathrm{M})$. Although this somewhat restricted the lower wavelength limit of data registering, a low concentration is mandatory to observe reversible thermal unfolding scans [15]. CD spectral data are reported as mean residue ellipticity, $[\theta]$, which was calculated as $[\theta]=$ $100 \theta /(C l)$; in this expression $\theta$ is the measured ellipticity 
in degrees, $C$ is the mean residue molar concentration (mean residue $M_{\mathrm{r}}=107.5$ ), and $l$ is the cell path length in centimeters.

\section{Thermal transitions}

Conformational changes induced by heating or cooling of yTIM were continuously monitored by following the ellipticity at $220 \mathrm{~nm}$ while temperature was varied at $2.0{ }^{\circ} \mathrm{C} \mathrm{min}^{-1}$. Samples $(\approx 0.19 \mu \mathrm{M})$ were placed in a 1.00 $\mathrm{cm}$ cell with a magnetic stirrer, and the temperature within the cell was registered by the external probe of the Peltier-type accessory. Refolding profiles were registered immediately after the unfolding transitions had been completed.

\section{Kinetics studies}

Unfolding kinetics tracings were registered by following ellipticity changes at $220 \mathrm{~nm}$, as described previously $[15,25]$. Unfolding was initiated by adding a small aliquot of concentrated TIM solution to a $1.00-\mathrm{cm}$ cell containing buffer equilibrated at the temperature selected for each experiment. Within the cell, the temperature reached $\pm 0.15{ }^{\circ} \mathrm{C}$ of the final equilibrium value in about $15 \mathrm{~s}$. The final protein concentration was $0.19 \mu \mathrm{M}$ in most cases. Essentially, the same procedure was used for monitoring changes in intrinsic fluorescence over time. In this case, experiments were carried out in a K2 spectrofluorometer (ISS, Champaign, IL), which had a Peltier accessory. Protein samples were excited at $292 \mathrm{~nm}$, and the light emitted at $318 \mathrm{~nm}$ was collected. Kinetic data were analyzed using a triple exponential decay equation:

$$
\begin{aligned}
y= & y_{0}+A_{1}\left[\exp \left(-\lambda_{1} t\right)-1\right]+A_{2}\left[\exp \left(-\lambda_{2} t\right)-1\right] \\
& +A_{3}\left[\exp \left(-\lambda_{3} t\right)-1\right]
\end{aligned}
$$

where $y$ is the physical observable monitored as a function of time $t$, and $y_{0}$ is the initial value of the observable. $A_{i}$ and $\lambda_{i}$ represent the observed amplitude and rate constant, respectively, for the $i$ th exponential phase. In some cases, only two exponential terms were required for satisfactory curve fitting.

In refolding experiments, yTIM samples were first subjected to unfolding for $10 \mathrm{~min}$ at $63.0^{\circ} \mathrm{C}$. Then, the temperature control of the $\mathrm{CD}$ spectrometer Peltier accessory was set to a value $4.0{ }^{\circ} \mathrm{C}$ below the temperature intended for the study of the refolding reaction $\left(42.0^{\circ} \mathrm{C}\right)$ to allow for fast cooling of the sample $\left(\approx 15{ }^{\circ} \mathrm{C} \min ^{-1}\right)$. The final temperature value $\left(42.0{ }^{\circ} \mathrm{C}\right)$ was entered into the cell-holder control when the solution in the cell was $0.5{ }^{\circ} \mathrm{C}$ above that value, and the $\mathrm{CD}$ signal was registered thereafter. Inside the cell, temperature came to equilibrium $\left( \pm 0.15{ }^{\circ} \mathrm{C}\right)$ in approximately $40 \mathrm{~s}$.

\section{Molecular dynamics simulations}

Molecular dynamics (MD) simulations were performed using GROMACS 4.5.4 software [26] with the GROMOS96 53A6 force-field [27]. The side-chain ionization states in the protein at the $\mathrm{pH}$ values simulated $(6.7,7.4$, and 8.0) were established using $p K_{a}$ values estimated with PROPKA [28]. Dimeric yTIM (PDB ID: 1YPI) was placed in the center of a periodic dodecahedral box with $10 \AA$ between the protein and the edge of the box. To simulate the solvent conditions at $\mathrm{pH} 6.7(7.4 ; 8.0)$, a total of $21,763(21,757 ; 21751)$ SPC water molecules, 12 $(16 ; 20)$ sodium ions, and $7(10 ; 12)$ chloride ions were needed to fill the box, neutralize the net protein charge, and reach the experimental ionic strength of $0.015 \mathrm{M}$ (0.022 M; $0.027 \mathrm{M})$.

Prior to MD simulations, the system was relaxed by energy minimization, followed by 100 ps of thermal equilibration under the position restraints of protein heavy atoms through a harmonic force constant of $1000 \mathrm{~kJ} \mathrm{~mol}^{-1} \mathrm{~nm}^{-1}$. MD simulation was performed using an NPT ensemble at $423 \mathrm{~K}$ and 1.0 bar for $100 \mathrm{~ns}$. A LINCS algorithm was applied to constrain the length of all covalent bonds [29], and a 2-fs time step was used. A cutoff of $1.0 \mathrm{~nm}$ was applied for short-range electrostatic and van der Waals interactions, while the longrange electrostatic forces were treated using the particle mesh Ewald method [30]. Two replicas were simulated at each solvent condition.

\section{Results and discussion}

\section{Unfolding-refolding thermal transitions}

Denaturation (unfolding) and renaturation (refolding) of yTIM were followed by continuous monitoring of the ellipticity $(220 \mathrm{~nm})$ under a constant heating or cooling rate of $2.0{ }^{\circ} \mathrm{C} \mathrm{min}{ }^{-1}$. Temperature scanning profiles recorded at three different $\mathrm{pH}$ values are shown in Fig. 1. These profiles display the hysteresis phenomenon previously observed for yTIM [15, 16, 25], which indicates that unfolding and refolding events occur under kinetic control at the imposed scanning rate $[14,15]$. It is clear that $\mathrm{pH}$ has an influence on the kinetic stability of the protein, because the apparent melting temperature is displaced to lower values at $\mathrm{pH}$ 8.5. Despite this $\mathrm{pH}$ effect, all the unfolding traces appear as sigmoid curves, with no evidence of stable intermediates. However, the total ellipticity change at pH 6.7 that takes place upon denaturation seems slightly larger (Fig. 1). It must be noted that the up-temperature scans in Fig. 1 were not allowed to proceed to higher temperatures to avoid reactions that make the process irreversible and thus decrease the extent of refolding on down-temperature scans [15].

In a different set of experiments, denatured samples of the enzyme were left to stand at $70{ }^{\circ} \mathrm{C}$ for $10 \mathrm{~min}$ to ensure that unfolding had been completed before their $\mathrm{CD}$ 


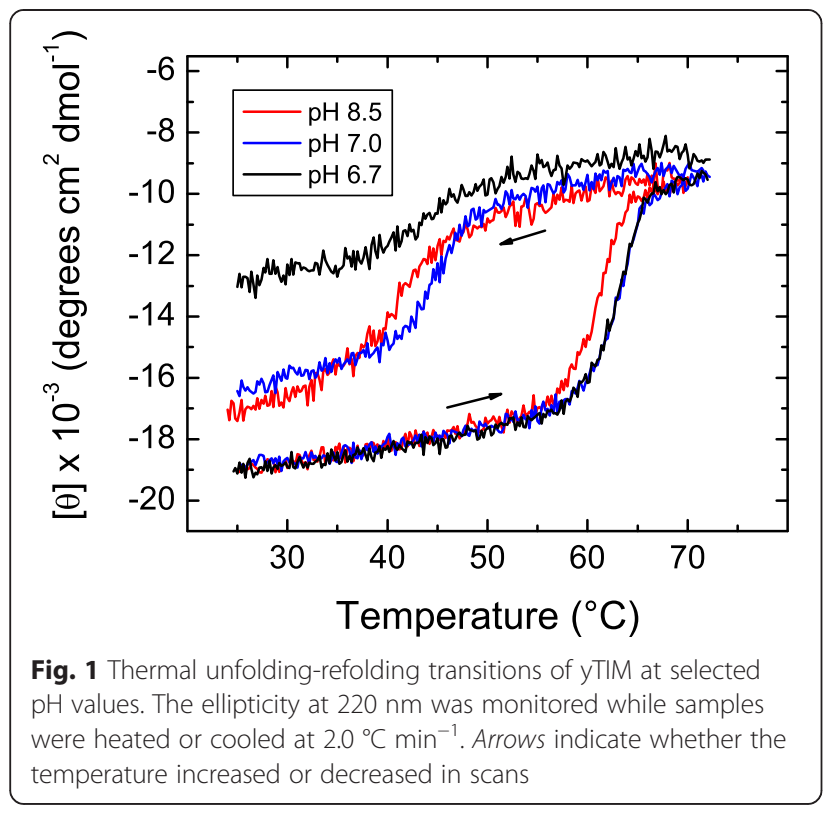

spectra were recorded. Spectra shown in Fig. 2 indicate that the native $\alpha / \beta$ secondary structure of yTIM is rather insensitive to $\mathrm{pH}$ and is largely lost upon heating at all $\mathrm{pH}$ values, as judged by the decrease in magnitude in the 208-222 nm region at high temperature (Fig. 2). However, the spectrum of heat-denatured yTIM shows striking changes as $\mathrm{pH}$ is varied. Above $\mathrm{pH} 8.0$, the spectral shape and signal magnitude of the denatured enzyme are typical of small and medium-size proteins (e.g., hen-egg lysozyme, ribonuclease $\mathrm{A}$, cytochrome $\mathrm{C}$, staphylococcal nuclease, cysteine proteinases) when unfolded at high

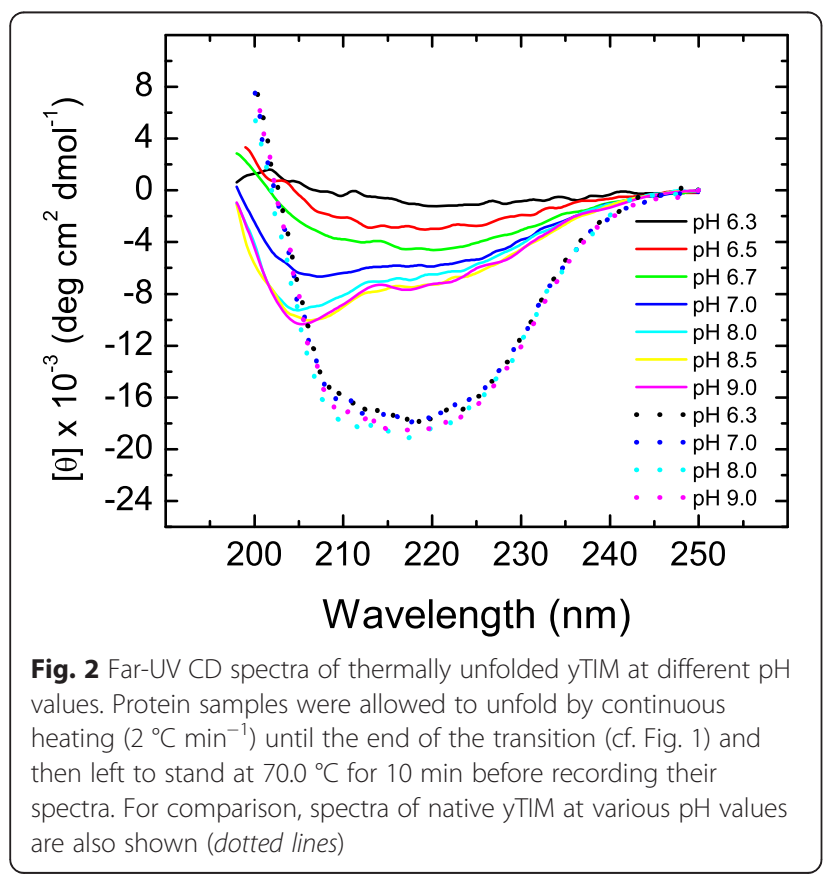

temperatures in the absence of denaturant agents (see, for example, CD spectra of native and thermally unfolded lysozyme in Additional file 2). This spectral type is characterized by a negative band of approximately $10 \times 10^{3} \mathrm{deg} \mathrm{cm} \mathrm{dmol}^{-1}$ centered at $202-204 \mathrm{~nm}$, along with a broad negative shoulder with magnitude around $5 \times 10^{3} \mathrm{deg} \mathrm{cm}^{2} \mathrm{dmol}^{-1}$ at longer wavelength $[31,32]$. Below pH 8.0, the spectra of heat-denatured yTIM progressively decrease in magnitude and acquire a shape typical of all- $\beta$ proteins [33], thus pointing to the presence of residual secondary structure in the denatured enzyme.

Regarding yTIM refolding in cooling-down scans, it is evident that this process becomes increasingly irreversible as the $\mathrm{pH}$ decreases below $\mathrm{pH}$ 7.0, as judged from the extent of recovery of native yTIM ellipticity shown in Fig. 1. To gain detailed information on the influence of $\mathrm{pH}$ in the unfolding and refolding events, further kinetic experiments were carried out.

\section{Unfolding kinetics}

Kinetic studies were carried out by monitoring the time course of ellipticity at $220 \mathrm{~nm}$. Experiments examining a large temperature interval were done at $\mathrm{pH} 8.0$ and 6.7, where the CD spectra of denatured yTIM showed distinct features. The results at $\mathrm{pH} 8.0$ indicate that at relatively high temperatures $\left(60.0^{\circ} \mathrm{C}\right.$ and above), the loss of secondary structure shows double exponential behavior (Fig. 3), with phases well separated on the time scale. Indeed, over a restricted time interval, a single exponential-decay equation can fit the experimental data reasonably well. Only when data were recorded over a long time did a second phase become readily apparent, but this phase had small amplitude. Nevertheless, at low temperature, triple exponential behavior was observed (Fig. 3). The fastest phase, which conveys a minor ellipticity change, occurred too fast for accurate assessment of the kinetic constant by the manual-mixing method (i.e., time constant of about 20 to $100 \mathrm{~s})$. This fast phase seems to be completely lost within the dead time in experiments at high temperature. Hereafter, the observed rate constants are referred to as $\lambda_{1}, \lambda_{2}$, and $\lambda_{3}$, in descending order of their magnitudes. Unfolding of yTIM at pH 6.7 showed similar behavior, with two and three kinetic phases at high and low temperature, respectively, as shown in Additional file 3.

$\mathrm{CD}$ spectra were recorded near the end of the unfolding process when the slowest phase was more than $98 \%$ complete (these experiments required recordings of kinetic data for more than nine hours in the case of low temperatures). The final spectra appeared nearly identical, notwithstanding the temperature at which the kinetics was studied (see Additional file 4 for results obtained at $\mathrm{pH}$ 8.0). Furthermore, at a given $\mathrm{pH}$, the spectral shape and magnitude observed at the end of unfolding were both similar to those illustrated in Fig. 2. In other 


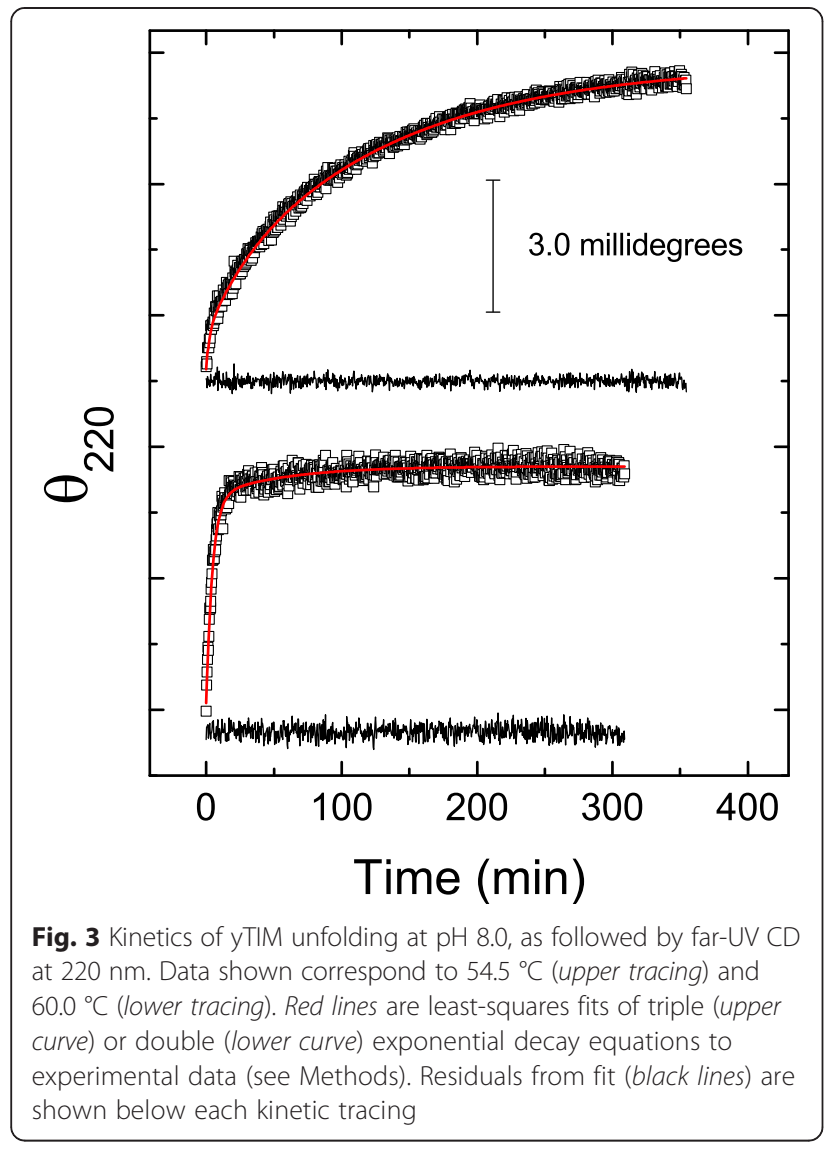

words, the final conformation achieved by the protein seems to be independent of the temperature (in the range studied), but is otherwise strongly affected by $\mathrm{pH}$.

The voltage applied to the phototube of the CD instrument, which is proportional to the absorbance, was simultaneously recorded. The measurements indicated that changes in ellipticity associated with the first two phases are accompanied by only small changes (5.0 \% or less) in the absorbance of the protein solution (see Additional file 5). Such small changes are known to occur due to alterations in the secondary and, to less extent, the tertiary structure of proteins and polypeptides [34]. However, a relatively large absorbance increment (approximately $10.0 \%$ of the protein absorbance) was linked to the slower CD-detected kinetic phase. It is likely that this apparent increment comes from the scattering of light by aggregates of unfolded protein molecules.

Monitoring of the denaturation kinetics by changes in the fluorescence intensity also showed that this is a complex process (Fig. 4) in which there is a progressive decrease of intensity (at the wavelength of maximum emission by native yTIM). Overall, comparison of the plots shown in Figs. 3 and 4 indicates that progressive loss of secondary structure upon denaturation is accompanied by a quenching of the fluorescence signal of

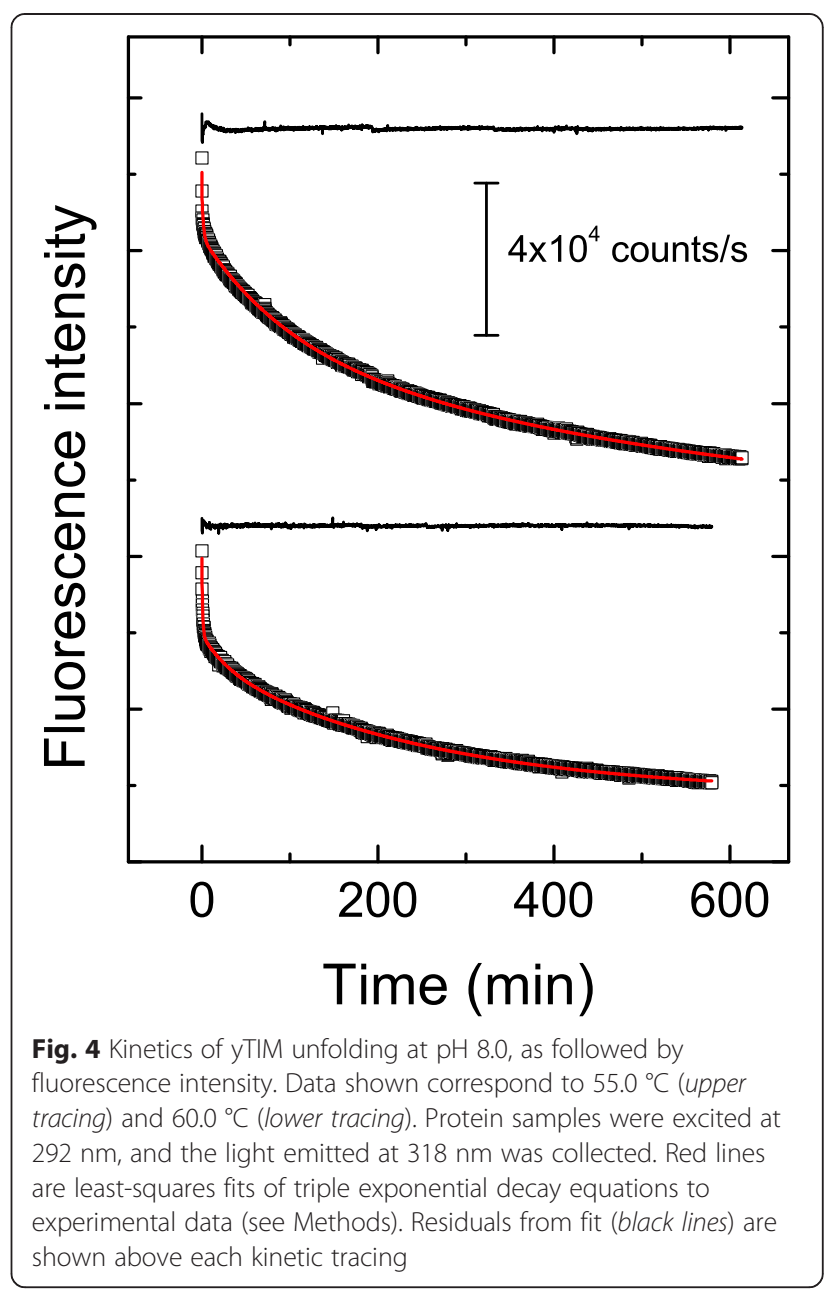

tryptophan residues, which in turn likely reflects either the exposure of these residues to the aqueous solvent or less constraint by the environment [35]. Notwithstanding the temperature, three exponential terms were required to fit fluorescence data. As in CD experiments, the first fluorescence-detected phase was too fast (time constant of about $25 \mathrm{~s}$ ) for an accurate determination of its rate constant. At low temperature $\left(55.0{ }^{\circ} \mathrm{C}\right)$, the rate constant for the second phase had a value similar to that of $\lambda_{2}$ from $\mathrm{CD}$ experiments (the two values differed by 50 $80 \%$ ). At 62.0 to $64.0{ }^{\circ} \mathrm{C}$, however, it was the first fluorescence-detected rate constant that was consistent with $\lambda_{2}$. Furthermore, the decrease in the fluorescence intensity extended over a much longer time than the change in ellipticity (i.e., the rate constant for the slowest phase was approximately three- to fourfold smaller when determined from fluorescence than from $\mathrm{CD}$ ). These markedly different values suggest that the slowest phase comprises several elementary steps that respond differently to the spectroscopic probes employed. For instance, formation of molecular aggregates can conceivably occur with little or no change in secondary 
conformation, but with an otherwise significant fluorescence quenching of tryptophan residues.

\section{Kinetic model for yTIM unfolding}

The simplest model accounting for the results obtained from $C D$ would be that of three sequential reactions (Scheme 1), with native and unfolded yTIM (N and $\mathrm{U}$, respectively) and two intermediate species (I and X):

In this model, each of the three $\lambda$ values determined from data analysis (eqn. 1) is identical to each one of the microscopic rate constants $k_{1}, k_{2}$, and $k_{3}$. As mentioned, neither the rate constant nor the amplitude of the faster phase could be accurately determined from experiments at the lowest temperatures studied. Moreover, this phase was apparently lost within the dead time of experiments performed at high temperature. Fortunately, because $k_{1}$ seems to be 15-20 times larger than $k_{2}$, the first kinetic step occurs on a much shorter time scale than the other steps and can be regarded as kinetically separated from the other events, at least in a first approximation. This implies that amplitudes $A_{2}$ and $A_{3}$ reflect changes involved solely with steps $\mathrm{I} \rightarrow \mathrm{X} \rightarrow \mathrm{U}$. Therefore, the kinetic model can be simplified to a two-step model (Scheme 2).

Equations describing the evolution in time of the fraction of each species are well known [36, 37]. By denoting the characteristic ellipticity of each species as $\theta_{\mathrm{I}}, \theta_{\mathrm{X}}$, and $\theta_{\mathrm{U}}$, it can be shown that (see Additional file 6):

$$
\left(\theta_{\mathrm{X}}-\theta_{\mathrm{I}}\right) /\left(\theta_{\mathrm{U}}-\theta_{\mathrm{I}}\right)=k_{3} / k_{2}-\left[\left(k_{3}-k_{2}\right) / k_{2}\right]\left[A_{2} /\left(A_{2}+A_{3}\right)\right]
$$

and

$$
\begin{aligned}
\left(\theta_{\mathrm{U}}-\theta_{\mathrm{X}}\right) /\left(\theta_{\mathrm{U}}-\theta_{\mathrm{I}}\right)= & -\left[\left(k_{3}-k_{2}\right) / k_{2}\right] \\
& \times\left[A_{3} /\left(A_{2}+A_{3}\right)\right]
\end{aligned}
$$

The two equations above were used to compute $\left(\theta_{\mathrm{X}}-\theta_{\mathrm{I}}\right) /$ $\left(\theta_{\mathrm{U}}-\theta_{\mathrm{I}}\right)$ and $\left(\theta_{\mathrm{U}}-\theta_{\mathrm{X}}\right) /\left(\theta_{\mathrm{U}}-\theta_{\mathrm{I}}\right)$, which give the ellipticity change as a fraction of the total change for each step in Scheme 2. The results indicate that the degree of unfolding occurring during the $\mathrm{I} \rightarrow \mathrm{X}$ step (normalized to a total unitary change) would vary from 0.35 to 0.70 over a temperature range of $11^{\circ} \mathrm{C}$ (Fig. 5). For the $\mathrm{X} \rightarrow \mathrm{U}$ step, a concomitant decrease in the degree of unfolding would take place. Admittedly, it seems unlikely that the conformation of intermediate species would vary so drastically within such a narrow temperature range. Alternatively, these results may point to the presence of an off-pathway intermediate or even different, parallel unfolding pathways with predominance that changes with temperature.

$$
N \stackrel{k_{1}}{\longrightarrow} I \stackrel{k_{2}}{\longrightarrow} X \stackrel{k_{3}}{\longrightarrow} U
$$

Scheme 1 Kinetic model for three sequential first-order reactions

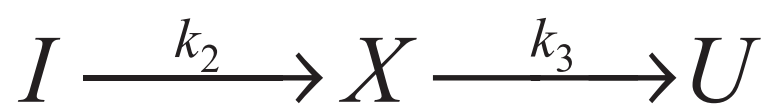

Scheme 2 Simplified kinetic model involving only two first-order steps

Temperature dependence of unfolding rate constants Further studies on the denaturation of the enzyme were also performed at other $\mathrm{pH}$ values but over a restricted temperature range to determine the activation parameters that control the temperature dependence of $k_{2}$ and $k_{3}$. Results for selected pH values are shown in Fig. 6 as Eyring plots, which agree with the well-known equation:

$$
\ln (k / T)=\ln E+\Delta S^{\ddagger} / R-\left(\Delta H^{\ddagger} / R\right)(1 / T)
$$

where $k$ is the rate constant for an elementary reaction, $T$ is the absolute temperature, $E$ stands for a preexponential factor, and $\Delta S^{\dagger}$ and $\Delta H^{\dagger}$ represent the activation entropy and enthalpy, respectively. Figure 6a shows that plots corresponding to $k_{2}$ follow linear trends when a narrow temperature range is considered. This linearity was observed before for yTIM and has been found for a large number of other proteins [15]. However, in the cases of $\mathrm{pH} 6.7$ and 8.0, at which larger temperature intervals were examined, Eyring plots appear slightly curved upwards in the low temperature region. This might be due to a shift between parallel unfolding pathways with different activation enthalpies [38]; that is, unfolding would switch from one predominant pathway to another as the temperature varies, in agreement with the interpretation mentioned for the change with

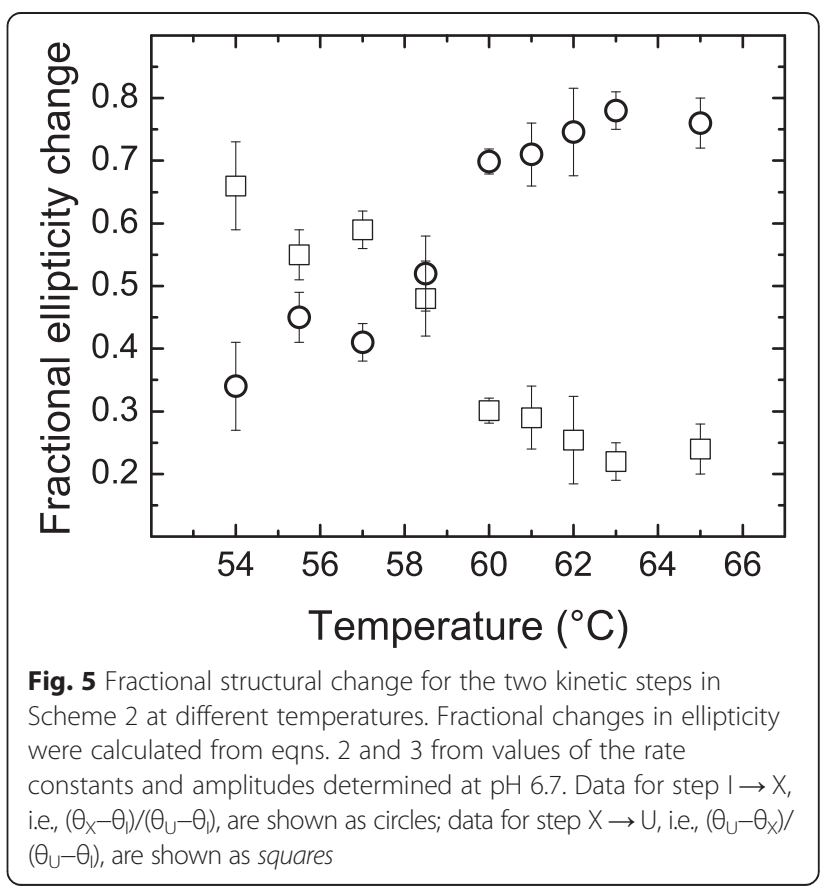



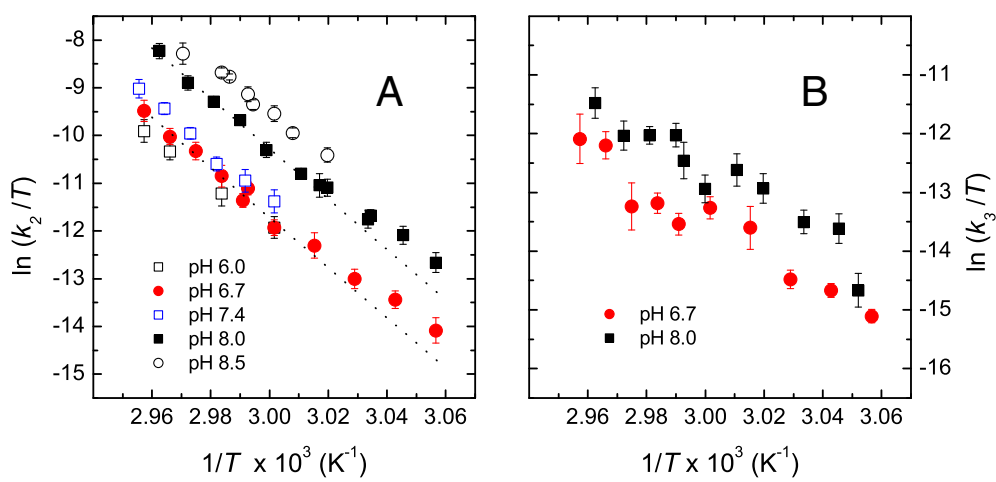

Fig. 6 Eyring plots for the rate constants $k_{2}(\mathbf{a})$ and $k_{3}(\mathbf{b})$ at selected $\mathrm{pH}$ values. Rate constants were determined from far-UV CD kinetic experiments. Dotted lines in (a) are linear fits performed with data corresponding to temperatures above $60.0^{\circ} \mathrm{C}$ for $\mathrm{pH} 6.7$ and 8.0

temperature of the computed degree of unfolding for step $\mathrm{I} \rightarrow \mathrm{X}$. However, a nonzero activation heat capacity cannot be ruled out as the origin of the curvature.

From Eyring plots, such as those in Fig. $6 \mathrm{a}, \Delta H_{2}^{\ddagger}$ was determined between $\mathrm{pH} 6.0$ and 8.5. It must be noted that values of $k_{2}$ were determined from data registered in a temperature region in which the unfolding degree accompanying step $\mathrm{I} \rightarrow \mathrm{X}$ remains relatively constant (i.e., from 60 to $65{ }^{\circ} \mathrm{C}$, cf. Fig. 5). Therefore, $k_{2}$ can be assigned to a single predominant pathway. Overall, the value of $\Delta H_{2}^{\dagger}$ was about $450 \mathrm{~kJ} \mathrm{~mol}^{-1}$ at $\mathrm{pH} 6.0-8.0$ and showed a slight decrease $(\approx 15 \%)$ at $\mathrm{pH} 8.5$ (data not shown). In contrast, Eyring plots for $k_{3}$ display a linear but ill-defined trend (Fig. 6b), suggesting that the slowest kinetic phase is indeed composed of several elementary steps. It is also seen that $k_{3}$ is much less temperature dependent than $k_{2}$.

The effect of $\mathrm{pH}$ on $k_{2}$ and $k_{3}$ was examined over a longer interval of $\mathrm{pH}$ values at constant temperature; $60.0{ }^{\circ} \mathrm{C}$ was chosen, because of the single apparent pathway at this temperature, and the unfolding process was slow enough to allow for determining the value of $k_{2}$ over an extended $\mathrm{pH}$ range. Results are shown in Fig. 7, which shows that $\mathrm{pH}$-induced changes in $k_{2}$ resemble the sigmoid titration curve for an ionizable group with an approximate $\mathrm{p} K_{a}$ of 8.5. Because this value of $\mathrm{p} K_{a}$ is close to that of a thiol group, it may be hypothesized that a cysteine residue is responsible for the behavior observed for $k_{2}$. In this regard, it has been proposed that Cys126, which is a residue that is conserved with the family of TIM enzymes, plays an important role in the stability of this protein [24]. In contrast, $k_{3}$ values showed no defined variation with $\mathrm{pH}$, again suggesting that the step $\mathrm{X} \rightarrow \mathrm{U}$ actually comprises multiple individual reactions.

\section{Refolding of yTIM}

As reported previously $[15,24,25]$, the kinetics of yTIM refolding at low protein concentration $(0.13-0.75 \mu \mathrm{M})$ and in a certain temperature range is slow enough to be monitored without resorting to fast temperature-jump techniques. By using the procedure described in the Methods section, we followed the recovery of secondary structure under two $\mathrm{pH}$ conditions. These studies were aimed at determining the effect of the residual nativelike structure of unfolded yTIM (which is clearly observed at $\mathrm{pH}$ 6.7) on the refolding ability of the enzyme. For this purpose, yTIM samples were allowed to unfold (in the cell of the CD instrument) for $10 \mathrm{~min}$ at $63.0{ }^{\circ} \mathrm{C}$. These conditions ensured ca. $85 \%(\mathrm{pH} 6.7)$ or $99 \%$ ( $\mathrm{pH}$ 8.0) unfolding, as judged by the ellipticity signal. After that, the protein solution was cooled to $42.0{ }^{\circ} \mathrm{C}$ to record the refolding reaction. Additional file 7 shows that at $\mathrm{pH} 6.7$ the enzyme refolds faster than at $\mathrm{pH}$ 8.0. In both cases, however, refolding tracings are adequately described by a second-order kinetics equation, as determined previously $[15,24]$.

To explore the effect of the residual structure on the reversibility of the unfolding process, samples of yTIM

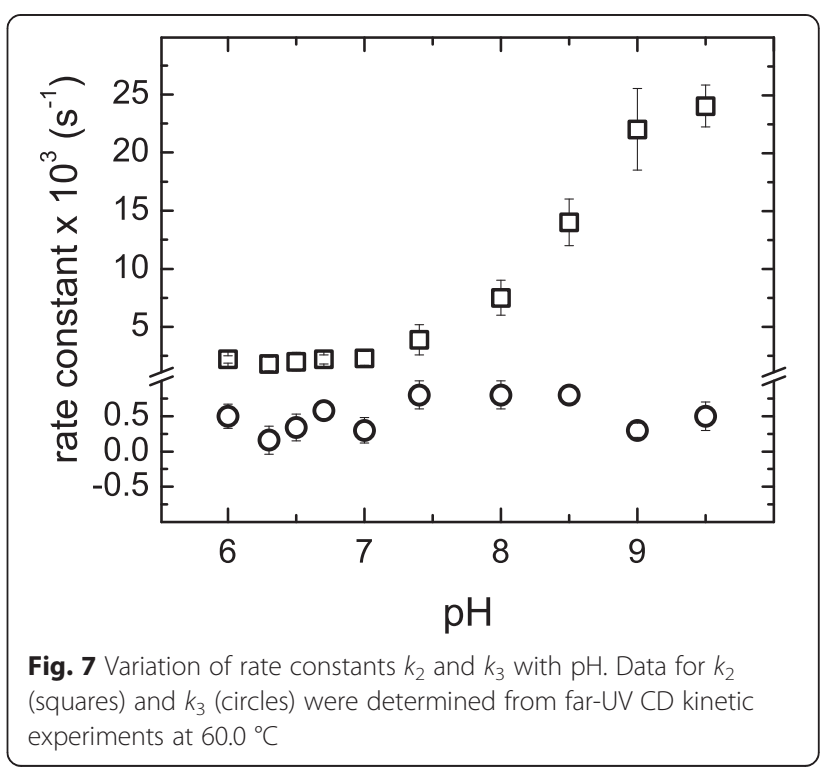




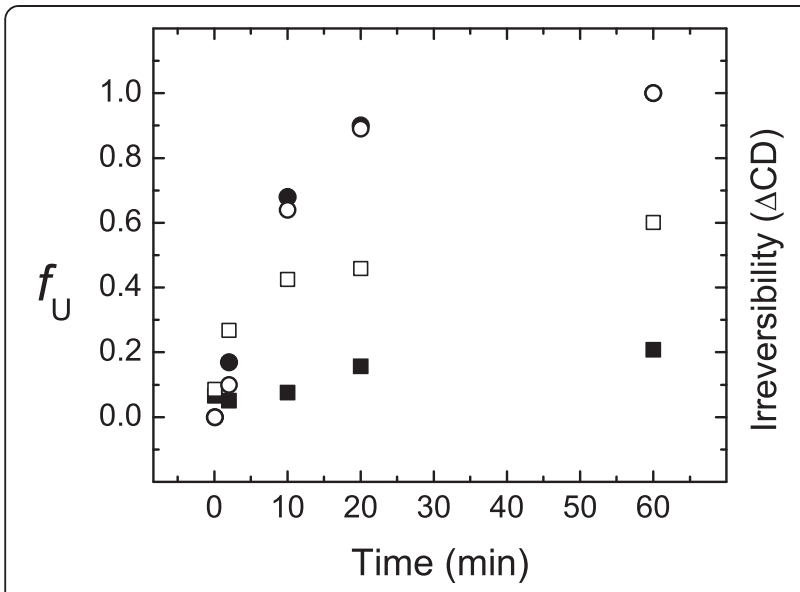

Fig. 8 Time course for the appearance of irreversibility on the unfolding of yTIM. Samples of YTIM were unfolded $\left(63.0^{\circ} \mathrm{C}\right)$ for different time spans, and then cooled to $25^{\circ} \mathrm{C}$ for recording of $\mathrm{CD}$ spectra. Irreversibility was then calculated as the difference in ellipticity $(220 \mathrm{~nm})$ between cooled-down samples and native yTIM, normalized to the ellipticity of the native protein. Irreversibility data are represented by open $(\mathrm{pH}$ 6.7) or solid ( $\mathrm{pH}$ 8.0) squares. Open $(\mathrm{pH}$ 6.7) and solid (pH 8.0) circles correspond to the fraction of unfolded protein, $f_{\mathrm{U}}$, which was calculated from eqn. $\mathrm{S} 3$ in Additional file 5

were unfolded for different time spans and then cooled to $25{ }^{\circ} \mathrm{C}$ to record $\mathrm{CD}$ spectra. As a quantitative indicator of irreversibility, the difference in ellipticity $(220 \mathrm{~nm})$ between cooled-down samples and native yTIM, normalized to the ellipticity of the native protein, was used. The results are shown in Fig. 8, together with the fractional values of $U\left(f_{\mathrm{U}}\right)$ in Scheme 2. Experimentally determined values of the kinetic constants $k_{2}$ and $k_{3}$ were used to calculate the time variation of $f_{\mathrm{U}}$ according to eqn.S3 in Additional file 6. An inspection of the plots in the figure makes it evident that irreversibility is more intense at $\mathrm{pH} 6.7$ than $\mathrm{pH} \mathrm{8.0,} \mathrm{as} \mathrm{expected} \mathrm{from} \mathrm{the} \mathrm{ther-}$ mal scan results (cf. Fig. 1). At the lower pH, however, irreversibility begins with early unfolding times and approximately parallels the formation of $f_{\mathrm{U}}$. In contrast, the onset of irreversibility at $\mathrm{pH} 8.0$ is delayed and thus appears as a late event in unfolding, which takes place after the final $U$ state becomes largely populated. This suggests again that the slowest $\mathrm{CD}$-detected kinetic phase does not represent an elementary step. Reactions that lead to irreversibility probably do not involve major changes in secondary conformation and are therefore silent in CD studies.

In summary, results from refolding studies indicate that yTIM refolds faster from the denatured state with residual structure, although such denatured state decreases the folding efficiency (i.e., the amount of native protein recovered upon refolding). Thus, it may be thought that under physiological conditions $(\mathrm{pH}$ near neutrality, $37^{\circ} \mathrm{C}$ ) the advantage of a fast folding process overcomes the difficulties posed by some degree of irreversibility. Furthermore, because irreversibility appears to be related to the time unfolded (denatured) YTIM stays at moderate to high temperatures [15], the problem of a low folding efficiency may be of less significance for mesophilic organisms such as Saccharomyces cerevisiae.

\section{Residual structure}

As mentioned, thermally denatured yTIM retains a high content of $\beta$ structure below pH 8.0 (see Fig. 2), which is implicated in reactions leading to irreversibility. This type of secondary structure has been found to be refractory to temperature in thermophilic and mesophilic proteins $[39,40]$, whereas in other instances, such as in apomyoglobin, $\beta$ structure appears to be formed at elevated temperature [31] as a result of $\alpha$-to- $\beta$ transitions [41]. Furthermore, molecular dynamics simulations have shown that certain all- $\alpha$ peptides, and even full-length proteins, may be transformed to all- $\beta$ structures $[42,43]$. We carried out preliminary MD simulations to investigate whether this method can reproduce the structural differences in denatured yTIM that were experimentally observed when $\mathrm{pH}$ is varied. Simulations run at $400 \mathrm{~K}$ for 100 ns showed that helixes are completely lost after $75 \mathrm{~ns}$, regardless of the $\mathrm{pH}$ value. Conversely, $\beta$-strands actually seem to be formed during the simulation, but they are slightly more abundant at $\mathrm{pH} 6.7$ than at $\mathrm{pH} 8.0$ (Additional file 8). Although preliminary, these results are encouraging, for they indicate that some regions in the polypeptide sequence of yTIM have a tendency to undergo $\alpha$-to- $\beta$ transitions. In contrast, the effect of $\mathrm{pH}$ does not appear to have been properly taken into account by the MD method used here, and thus deserves to be studied further.

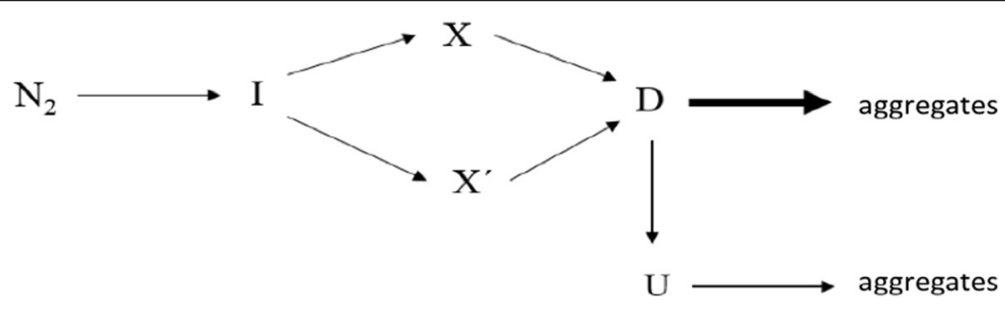

Scheme 3 Proposed model for yTIM unfolding 


\section{Conclusions}

Two experimental approaches were used to study the influence of $\mathrm{pH}$ on the temperature-induced unfolding of yeast triosephosphate isomerase (yTIM). Temperature-scan experiments showed that unfolding profiles (monitored by CD) appear as monophasic transitions, with no evidence of intermediate species. $\mathrm{pH}$ was found to affect the kinetic stability of the protein based on shifts in the melting temperature $\left(T_{m}\right)$. Furthermore, below $\mathrm{pH}$ 8.0, CD spectra of heat-denatured yTIM gradually changed in shape to look like those for proteins rich in $\beta$-strands, but otherwise, the unfolded protein became prone to aggregate.

Despite the apparent simplicity of thermal profiles, kinetic studies performed at constant temperature clearly showed the presence of up to three kinetic phases, irrespective of $\mathrm{pH}$ (i.e., at high temperatures, the fastest phase was completely lost within the experimental dead time). Because the relative values of the kinetic constants suggested that the fastest phase is indeed decoupled from the other two, we analyzed the kinetic constants and amplitudes of the two slowest phases according to a two-step sequential mechanism. Results from the analysis, however, pointed to a more complex actual mechanism, such as one that involves parallel pathways. The temperature dependence of the rate constants appears to lend some evidence to this proposal. A simple model for yTIM unfolding that accounts for the information summarized above is shown in Scheme 3, where $\mathrm{N}_{2}$ stands for the native dimer, I and $\mathrm{X}$ represent partially unfolded intermediates, and $\mathrm{D}$ and $\mathrm{U}$ are used to symbolize, respectively, the denatured form with $\beta$-strand residual structure and the thermally unfolded state of yTIM.

In summary, it was shown that the temperatureinduced denaturation of yTIM reveals itself as a complex process when followed for a long time and over an ample temperature range. Further investigation over a wide $\mathrm{pH}$ range showed that the kinetic stability of yTIM responds to the titration of an ionizable group with $p K_{a} \approx 8.5$. Refolding studies, on the other hand, indicated that the refolding ability of the unfolded protein decreases under $\mathrm{pH}$ conditions that favor the formation of residual, $\beta$-strand-like structures in heat-denatured yTIM, even though refolding is faster under such conditions. Moreover, most of the reactions leading to irreversibility occur late in the unfolding process and are not detected by CD. Finally, as demonstrated in molecular dynamics simulations, yTIM unfolding shows $\alpha$-to- $\beta$ transition behavior, albeit with no discrimination of the experimentally observed $\mathrm{pH}$ effect.

\section{Additional files}

Additional file 1: Mass Spectrum of isolated yTIM. (PDF 72 kb)
Additional file 2: Native and thermally unfolded hen-egg lysozyme CD spectra. (PDF $67 \mathrm{~kb}$ )

Additional file 3: Kinetics of yTIM unfolding at $\mathrm{pH}$ 6.7, as followed by CD. (PDF $210 \mathrm{~kb}$ )

Additional file 4: Far-UV CD spectra of yTIM near the end of the unfolding kinetics process. (PDF $89 \mathrm{~kb}$ )

Additional file 5: Kinetics of yTIM unfolding detected by light absorption. (PDF $142 \mathrm{~kb}$ )

Additional file 6: Derivation of eqns. 2 and 3 in text. (PDF 113 kb)

Additional file 7: Refolding kinetics of yTIM as followed by CD.

(PDF $133 \mathrm{~kb}$ )

Additional file 8: Molecular dynamics simulations of yTIM unfolding. (PDF $113 \mathrm{~kb}$ )

\section{Abbreviations}

yTIM: Triosephosphate isomerase from yeast (Saccharomyces cerevisiae); CD: Circular dichroism; MD: Molecular dynamics.

\section{Competing interests}

The authors declare that they have no competing interests.

\section{Authors' contributions}

ALP and MCC carried out most of the spectroscopic experiments, performed part of the data analysis, and participated in the experimental design and interpretation of results. GGR helped with the experiments, data analysis, and interpretation of results. RAZ performed the molecular dynamics simulations, participated in the interpretation of results, and helped in drafting the manuscript. AHA conceived of the study, participated in its design, carried out a major part of data analysis, and drafted the manuscript. All authors read and approved the final manuscript.

\section{Acknowledgements}

This work was funded in part by CONACYT, México (SEP-CONACYT2007-80457, and SEP-CONACYT 2012-181049). ALP received a doctoral fellowship from CONACYT, México (208217). The authors thank Dr. Ponciano García-Gutiérrez (Laboratorio Interdivisional de Espectrometría de Masas, UAM-Iztapalapa) for obtaining the mass spectrum of yTIM.

\section{Author details}

1Área de Biofisicoquímica, Departamento de Química, Universidad Autónoma Metropolitana-Iztapalapa, San Rafael Atlixco 186, Iztapalapa D.F. 09340, Mexico. ${ }^{2}$ Departamento de Bioquímica, Facultad de Medicina, Universidad Nacional Autónoma de México, Coyoacán D.F. 04510, Mexico.

Received: 24 March 2015 Accepted: 19 August 2015

Published online: 03 September 2015

\section{References}

1. Sánchez IE, Kiefhaber T. Evidence for sequential barriers and obligatory intermediates in apparent two-state protein folding. J Mol Biol. 2003;325:367-76.

2. Ferguson N, Fersht A. Early events in protein folding. Curr Op Struc Biol. 2003;13:75-81.

3. Kamagata K, Arai M, Kuwajima K. Unification of the folding mechanisms of non-two-state and two-state proteins. J Mol Biol. 2004;339:951-65.

4. Tsong TY. Detection of three kinetic phases in the thermal unfolding of ferricytochrome c. Biochemistry. 1973;12:2209-14.

5. Baldwin RL. On-pathway versus off-pathway folding intermediates. Folding \& Design. 1996;1:R1-8.

6. Aghera N, Udgaonkar JB. The utilization of competing unfolding pathways of monellin is dictated by enthalpic barriers. Biochemistry. 2013;52:5770-9.

7. Travaglini-Allocatelli C, Ivarsson Y, Jemth P, Gianni S. Folding and stability of globular proteins and implications for function. Curr Opin Struct Biol. 2009;19:3-7.

8. Wong KB, Clarke J, Bond CJ, JoseÂ Luis Neira J, Freund SMV, Fersht AR, et al. Towards a complete description of the structural and dynamic properties of the denatured state of barnase and the role of residual structure in folding. J Mol Biol. 2000;296:1257-82. 
9. Pearce MC, Cabrita LD, Rubin H, Gore MG, Bottomley SP. Identification of residual structure within denatured antichymotrypsin: implications for serpin folding and misfolding. Biochem Biophys Res Commun. 2004;324:729-35.

10. Sawano M, Yamamoto H, Ogasahara K, Kidokoro S, Katoh S, Ohnuma T, et al. Thermodynamic basis for the stabilities of three CutA1s from Pyrococcus horikoshii, Thermus thermophilus, and Oryza sativa, with unusually high denaturation temperatures. Biochemistry. 2008;47:721-30.

11. Vázquez-Pérez AR, Fernández-Velasco DA. Pressure and denaturants in the unfolding of triosephosphate isomerase: the monomeric intermediates of the enzymes from Saccharomyces cerevisiae and Entamoeba histolytica. Biochemistry. 2007:46:8624-33.

12. Shirley BA. Urea and guanidine hydrochloride denaturation curves. In: Shirley BA, editor. Protein Stability and Folding. Theory and Practice. Totowa, NJ: Humana Press; 1995. p. 177-90.

13. Blancas-Mejía LM, Tischer A, Thompson JR, Tai J, Wang L, Auton M, et al. Kinetic control in protein folding for light chain amyloidosis and the differential effects of somatic mutations. J Mol Biol. 2014:426:347-61.

14. Dragan Al, Potekhin SA, Sivolob A, Lu M, Privalov PL. Kinetics and thermodynamics of the unfolding and refolding of the three-stranded Rhelical coiled coil, Lpp-56. Biochemistry. 2004:43:14891-900.

15. Benítez-Cardoza CG, Rojo-Domínguez A, Hernández-Arana A. Temperatureinduced denaturation and renaturation of triosephosphate isomerase from Saccharomyces cerevisiae: evidence of dimerization coupled to refolding of the thermally unfolded protein. Biochemistry. 2001;40:9049-58.

16. Samanta M, Banerjee M, Murthy MRN, Balaram H, Balaram P. Probing the role of the fully conserved Cys 126 in triosephosphate isomerase by sitespecific mutagenesis - distal effects on dimer stability. FEBS Journal. 2011;278:1932-43.

17. Pan H, Raza AS, Smith DL. Equilibrium and kinetic folding of rabbit muscle triosephosphate isomerase by hydrogen exchange mass spectrometry. J Mol Biol. 2004;336:1251-63.

18. Guzman-Luna V, Garza-Ramos G. The folding pathway of glycosomal triosephosphate isomerase: Structural insights into equilibrium intermediates. Proteins. 2012;80:1669-82.

19. Mixcoha-Hernández E, Moreno-Vargas LM, Rojo-Domínguez A, BenítezCardoza CG. Thermal-unfolding reaction of triosephosphate isomerase from trypanosoma cruzi. Protein J. 2007;26:491-8.

20. Cabrera N, Hernández-Alcántara G, Mendoza-Hernández G, Gómez-Puyou A Perez-Montfort R. Key residues of loop 3 in the interaction with the interface residue at position 14 in triosephosphate isomerase from Trypanosoma brucei. Biochemistry. 2008;47:3499-506.

21. Dhaunta N, Arora K, Chandrayan SK, Guptasarma P. Introduction of a thermophile-sourced ion pair network in the fourth beta/alpha unit of a psychophile-derived triosephosphate isomerase from Methanococcoides burtonii significantly increases its kinetic thermal stability. Biochim Biophys Acta. 1834;2013:1023-33

22. Vázquez-Contreras E, Zubillaga RA, Mendoza-Hernández G, Costas M, Fernández-Velasco DA. Equilibrium unfolding of yeast triosephosphate isomerase: a monomeric intermediate in guanidine- $\mathrm{HCl}$ and two-state behavior in urea. Protein Pept Lett. 2000;7:57-64.

23. Rozacky EE, Sawyer TH, Barton RA, Gracy RW. Studies of human triosephosphate isomerase: isolation and properties of the enzyme from erythrocytes. Arch Biochem Biophys. 1971;146:312-20.

24. González-Mondragón E, Zubillaga RA, Saavedra E, Chánez-Cárdenas ME, Pérez-Montfort R, Hernández-Arana A. Conserved cysteine 126 in triosephosphate isomerase is required not for enzymatic activity but for proper folding and stability. Biochemistry. 2004;43:3255-63.

25. Reyes-López CA, González-Mondragón E, Benítez-Cardoza CG, ChánezCárdenas ME, Cabrera N, Pérez-Montfort R, et al. The conserved salt bridge linking two C-terminal b/a units in homodimeric triosephosphate isomerase determines the folding rate of the monomer. Proteins. 2008;72:972-9.

26. Hess B, Kutzner C, van der Spoel D, Lindahl E. GROMACS 4: Algorithms for highly efficient, load-balanced, and scalable molecular simulation. J Chem Theory Comput. 2008;4:435-47.

27. Oostenbrink C, Villa A, Mark AE, van Gunsteren WF. A biomolecular force field based on the free enthalpy of hydration and solvation: the GROMOS force-field parameter sets 53A5 and 53A6. J Comput Chem. 2004;25:1656-76.

28. Li H, Robertson AD, Jensen JH. Very fast empirical prediction and interpretation of protein pKa values. Proteins. 2005;61:704-21.

29. Hess B, Bekker H, Berendsen HJC, Fraaije JGEM. LINCS: a linear constraint solver for molecular simulations. J Comput Chem. 1997;18:1463-72.
30. Darden T, York D, Pedersen L. Particle mesh Ewald: An $N^{*} \log (N)$ method for Ewald sums in large systems. J Chem Phys. 1993;98:10089-92.

31. Privalov PL, Tiktopulo El, Venyaminov SY, Griko YV, Makhatadze GI, Khechinashvili NN. Heat capacity and conformation of proteins in the denatured state. J Mol Biol. 1989;205:737-50

32. Arroyo-Reyna A, Hernández-Arana A. The thermal unfolding of stem bromelain is consistent with an irreversible two-state model. Biochim Biophys Acta. 1995;1248:123-8.

33. Manavalan P, Johnson WC. Sensitivity of circular dichroism to protein tertiary structure class. Nature. 1983;305:831-2.

34. Van Holde KE, Johnson WC, Ho PS. Principles of Physical Biochemistry. New Jersey: Prentice Hall International; 1998.

35. Campbell ID, Dwek RAR. Biological Spectroscopy. Menlo Park, CA: The Benjamin/Cummings Publishing Company; 1984

36. Gutfreund H. Kinetics for the Llife Sciences. Receptors, Transmitters and Catalysts. Cambridge, UK: Cambridge University Press; 1995.

37. Szabo ZG. Kinetic characterization of complex reaction systems. In: Banford $\mathrm{CH}$, Tipper CFH, editors. Comprehensive Chemical Kinetics. Volume 2. Amsterdam: Elsevier; 1969. p. 1-80.

38. Zaman MH, Sosnick TR, Berry RS. Temperature dependence of reactions with multiple pathways. Phys Chem Chem Phys. 2003;5:2589-94.

39. Toledo-Núñez C, López-Cruz Jl, Hernández-Arana A. Thermal denaturation of a blue-copper laccase: Formation of a compact denatured state with residual structure linked to $\mathrm{pH}$ changes in the region of histidine protonation. Biophys Chem. 2012;167:26-32.

40. Ausili A, Scire A, Damiani E, Zolese G, Bertoli E, Tanfani F. Temperatureinduced molten globule-like state in human R1-acid glycoprotein: An infrared spectroscopic study. Biochemistry. 2005:44:15997-6006.

41. Fabiani E, Stadler AM, Madern D, Koza MM, Tehei M, Hirai M, et al. Dynamics of apomyoglobin in the a-to- $\beta$ transition and of partially unfolded aggregated protein. Eur Biophys J. 2009;38:237-44.

42. GC JB, Bhandari YR, Gerstman BS, Chapagain PP. Molecular dynamics investigations of the a-helix to $\beta$-barrel conformational transformation in the RfaH transcription factor. J Phys Chem B. 2014;118:5101-8.

43. Kaur H, Sasidhar YU. Molecular dynamics study of an insertion/duplication mutant of bacteriophage T4 lysozyme reveals the nature of a- $\beta$ transition in full protein context. Phys Chem Chem Phys. 2013;15:7819-30.

\section{Submit your next manuscript to BioMed Central and take full advantage of:}

- Convenient online submission

- Thorough peer review

- No space constraints or color figure charges

- Immediate publication on acceptance

- Inclusion in PubMed, CAS, Scopus and Google Scholar

- Research which is freely available for redistribution

Submit your manuscript at www.biomedcentral.com/submit
C BioMed Central 